\title{
Evaluation of optical parameters of quasi-parallel plates with single-frame interferogram analysis methods
}

\author{
Zofia Sunderland, Krzysztof Patorski, Krzysztof Pokorski \\ Institute of Micromechanics \& Photonics, Warsaw University of Technology, 8 Sw. A. Boboli St., 02-525 Warsaw
}

Received May 15, 2012; accepted June 25, 2012; published June 30, 2012

\begin{abstract}
The surface flatness of transparent plates is frequently tested in Fizeau and Twyman-Green interferometers. In the case of quasi-parallel plates, however, the common problem is additional reflection from the plate rear surface and three-beam interference. Our new method of deriving shape and optical thickness variations of the plate requires recording two interferograms: a two-beam interferogram without a reference beam and a three-beam interference one. The images are processed by using single-frame techniques only. The proposed method does not require modification of the sample, sophisticated equipment or complicated data analysis.
\end{abstract}

The Fizeau and Twyman-Green interferometers are the most frequently used setups for optical element flatness testing. To achieve high accuracy of measurements some conditions must be fulfilled. The most important one is processing two beam interference patterns with perfectly cosinusoidal fringes. No additional reflections, no parasitic fringes are allowed. But in the case of quasiparallel plates with high quality of both surfaces the interference of more than two beams arises. The beams are reflected from the plate front and rear surfaces and the reference flat. Unwanted parasitic intensity distributions modulate the two-beam interferogram of the plate front surface [1] and make the application of phase shifting methods for automatic fringe pattern analysis [2] inefficient. Several approaches to suppress unwanted fringe modulations were proposed, but they all require either interference into a sample, or sophisticated equipment and complicated data analysis [3].

In this paper we propose the algorithm to calculate the shape of plate front and back surfaces under the assumption of homogeneous refractive index distribution $[1,3]$; it simplifies the evaluation process of optical plate parameters without resorting to the temporal phase shifting method [1, 3]. The two-beam interferogram intensity distribution recorded without a reference beam (with the information on the plate optical thickness variations) is subtracted from the three-beam interference pattern. This operation enables access to an interesting synthetic fringe pattern which can be treated, under some assumptions related to optical plate parameters and the

\footnotetext{
*E-mail: z.sunderland@mchtr.pw.edu.pl
}

interferometer reference beam tilt angle, as the sum of two separate intrinsic interferograms carrying separate information on the front and back surface shape of the plate under test. In this way, comprehensive information on all plate parameters can be obtained. Phase calculations from fringe patterns, at various evaluation stages, are performed using single frame processing methods, e.g., vortex (Hilbert) transformation [4] and continuous wavelet transformation [5]. Although the accuracy of single-frame methods is not as high as that of multi-frame temporal phase shifting, it is acceptable for preliminary testing and/or lower accuracy requirements.

Our method will involve a Twyman-Green interferometer. Tilting its reference beam mirror for appropriate separation of the component fringe patterns, does not introduce retracing errors as it would in Fizeau interferometry. Additionally, screening off the reference beam mirror to record the plate-only two-beam interferogram is much easier than removing the reference master in a Fizeau interferometer. Nevertheless, our proposal can be extended to the latter case as well.

The intensity distribution of the three-beam interference pattern created in a Twyman-Green interferometer while testing a quasi-parallel optical plate, Fig. 1(a), can be written as $[6,7]$ :

$$
\begin{aligned}
& I_{f b r}=A_{r}{ }^{2}+A_{f}{ }^{2}+A_{b}{ }^{2}+2 A_{f} A_{b} \cos \left(\theta_{f}-\theta_{b}\right) \\
& +2 A_{f} A_{r} \cos \left(\theta_{f}-\theta_{r}\right)+2 A_{r} A_{b} \cos \left(\theta_{r}-\theta_{b}\right)
\end{aligned}
$$

where, $A_{r}, A_{f}, A_{b}$ are the amplitudes and $\theta_{r}, \theta_{f}, \theta_{b}$ are the phases of the three beams, respectively. For notation brevity the spatial $(\mathrm{x}, \mathrm{y})$ dependence of all terms has been omitted. The two-beam interferogram formed by the beams reflected from the front and back surfaces of the plate, Fig. 1(b), can be described as:

$$
I_{f b}=A_{b}^{2}+A_{f}^{2}+2 A_{f} A_{b} \cos \left(\theta_{f}-\theta_{b}\right)
$$

Subtracting intensity distributions of the two-beam interferogram and the reference beam from the three-beam interferogram intensity pattern leads to the intensity distribution, Fig. 1(c), given by the following equation: 


$$
\begin{aligned}
& I_{f b r}-I_{f b}-A_{r}^{2}=2 A_{f} A_{r} \cos \left(\theta_{f}-\theta_{r}\right)+2 A_{b} A_{r} \cos \left(\theta_{b}-\theta_{r}\right) \\
& =4 A_{f} A_{r} \cos \left(\frac{\theta_{f}+\theta_{b}}{2}-\theta_{r}\right) \cdot \cos \left(\frac{\theta_{f}-\theta_{b}}{2}\right)
\end{aligned}
$$

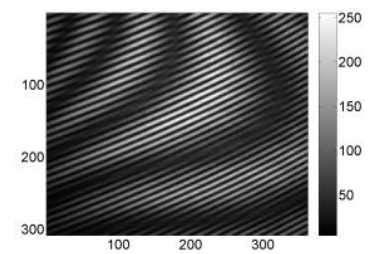

(a)

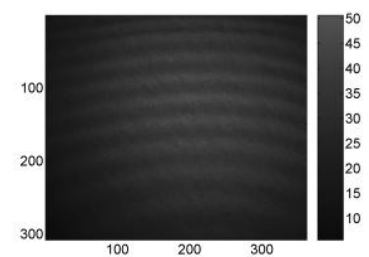

(c)

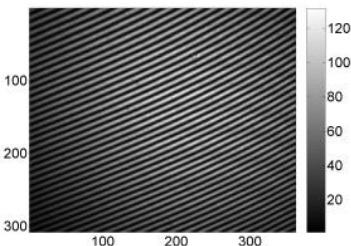

(b)

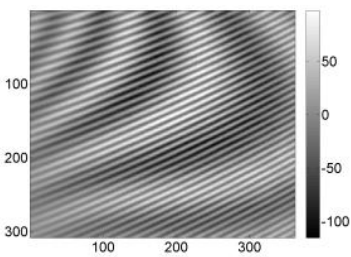

(d)
Fig. 1. (a) The three-beam interferogram $I_{f b r}$. (b) The two-beam interferogram $I_{f b}$. (c) The intensity distribution in the reference beam. (d) The intensity distribution given by Eq. (3).

Because of a glass plate protecting the CCD matrix, quasihorizontal, slightly bent parasitic fringes can be observed in Figs. 1(a)-1(c).

The intensity distribution, Eq. (3), may be interpreted as a sum of two fringe families: $2 A_{f} A_{r} \cos \left(\theta_{f}-\theta_{r}\right)$ and $2 A_{b} A_{r} \cos \left(\theta_{b}-\theta_{r}\right)$ or owing to trigonometric identities and the assumption of equal amplitudes of all beams, as a product of two other fringe families: $M 1=\cos \left(\frac{\theta_{f}+\theta_{b}}{2}-\theta_{r}\right)$ and $M 2=\cos \left(\frac{\theta_{f}-\theta_{b}}{2}\right)$.

In this paper we present two methods of processing the synthetic three-beam interferogram, Eq. (3). The first one, a multi-step method, starts with phase $\theta_{f}-\theta_{b}$ calculation using the vortex transform [4], see Fig. 2.

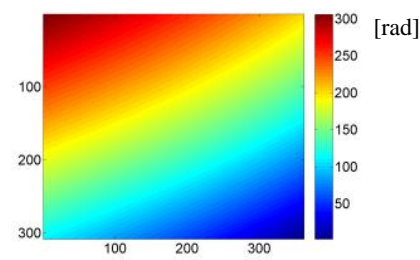

Fig. 2. Phase distribution $\theta_{f}-\theta_{b}$ calculated from the two-beam interference pattern using the vortex transform method.

Having the phase map $\theta_{f}-\theta_{b}$ the synthetic fringe pattern
$M 2=\cos \left(\frac{\theta_{f}-\theta_{b}}{2}\right)$, Fig. 3(a), may be created. In the next step the intensity distribution expressed by Eq. (3) is divided by $M 2$ what results in the synthetic fringe pattern $M 1$, shown in Fig. 3(b) after normalization. The points in which division by values close to zero took place are masked and then the normalization algorithm assigns appropriate values to them.

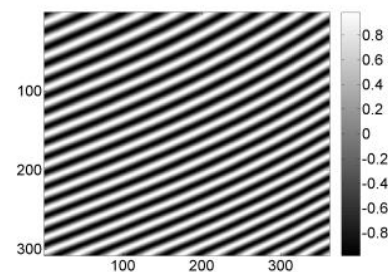

(a)

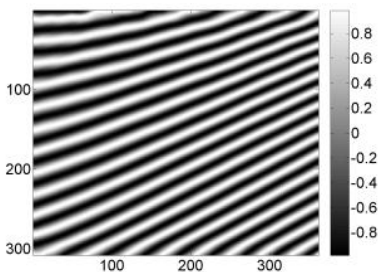

(b)
Fig. 3. (a) The synthetic two-beam interferogram M2. (b) The synthetic two-beam interferogram $M 1$.

Once again the vortex transform method is used to retrieve the phase map from a single image $M 1$, Fig. 4 (b).

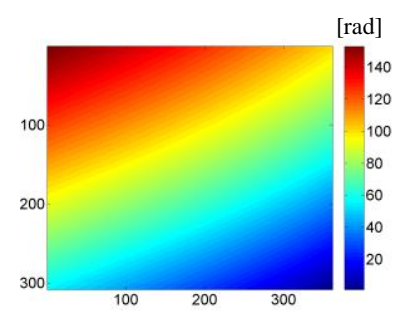

(a)

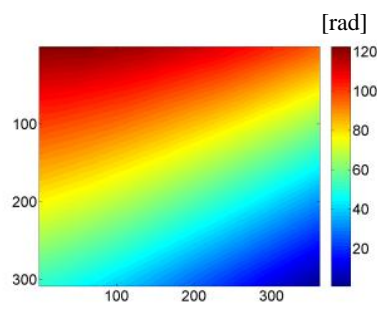

(b)
Fig. 4. The phase distributions of (a) $M 2$ and (b) $M 1$ computed using the VT technique.

Phase maps $\frac{\theta_{f}-\theta_{b}}{2}$ and $\frac{\theta_{f}+\theta_{b}}{2}-\theta_{r}$, Fig. 4, allow reconstructing the front and rear surface shapes with respect to the reference surface. Their sum and difference lead to searched phase distributions:

$$
\begin{aligned}
& \frac{\theta_{f}+\theta_{b}}{2}-\theta_{r}+\frac{\theta_{f}-\theta_{b}}{2}=\theta_{f}-\theta_{r} \\
& \frac{\theta_{f}+\theta_{b}}{2}-\theta_{r}-\frac{\theta_{f}-\theta_{b}}{2}=\theta_{b}-\theta_{r}
\end{aligned}
$$

The calculation results are presented in Fig. 5. 


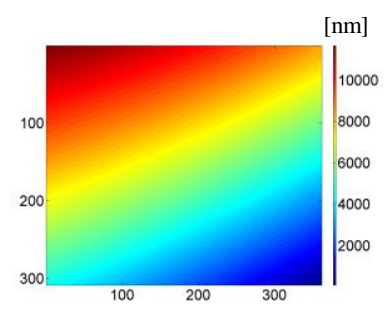

(a)

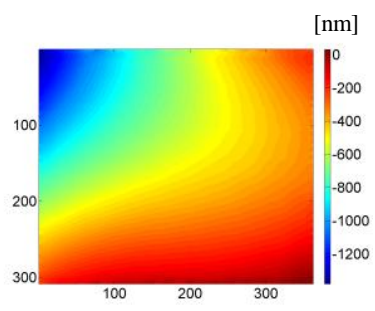

(b)
Fig. 5. Rescaled phase maps (a) $\theta_{f}-\theta_{r}$ and (b) $\theta_{b}-\theta_{r}$ showing the shape of plate front and back surfaces computed using VT.

The second method of analyzing the intensity pattern expressed by Eq. (3) applies the two-dimensional continuous wavelet transform (2D CWT) [5,8]. If space and frequency separation of fringe families is ensured, so that the lowest frequency of one of the fringe patterns forming the image in Fig. 1(d) is higher than the highest frequency of the second fringe family (the condition fulfilled in Fig. 1(d)), then they can be separated by using the 2D CWT. To get both fringe patterns separated and calculate phase maps from them, Fig. 6, the input image must be processed twice.

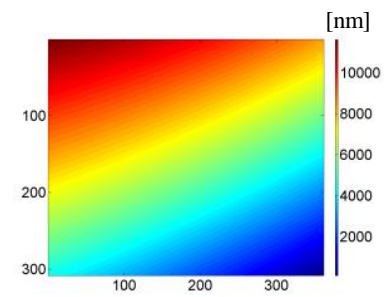

(a)

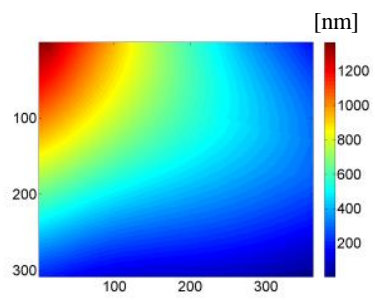

(b)
Fig. 6. Rescaled phase maps (a) $\theta_{f}-\theta_{r}$ and (b) $\theta_{b}-\theta_{r}$ showing the shape of plate front and back surfaces computed using 2D CWT.

The shape of the plate front surface was also computed using the temporal phase shifting (TPS) method which can serve as a reference technique (the most accurate method of fringe pattern analysis requiring registration of a few images precisely shifted in phase [2]). The reflection from the plate back surface was suppressed with the help of a special lacquer, so that analysed images were two-beam interferograms. The TPS result was compared with the two phase maps obtained using the 2D CWT and the multi-step VT analysis methods, Fig. 7.

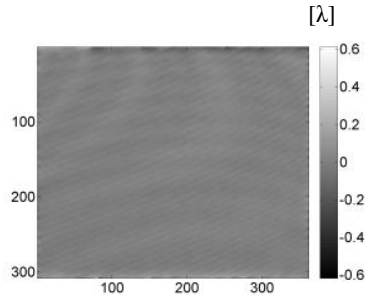

RMS: $0.0283 \lambda$

PV: $\quad 0.6282 \lambda$

(a)

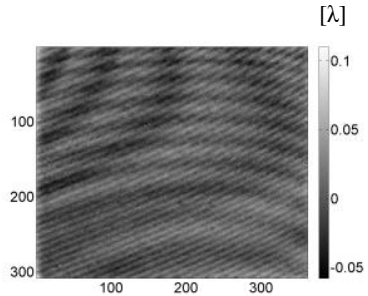

RMS: $0.0159 \lambda$

PV: $\quad 0.1114 \lambda$

(b)
Fig. 7. The difference between rescaled phase distributions obtained using (a) TPS and VT, and (b) TPS and 2D CWT methods.

The values of RMS and PV errors lie in the range typical for single-frame techniques of interferogram analysis and are acceptable for preliminary tests. In the multi-step VT method it is impossible to avoid error propagation, so the values of RMS and PV are higher. But its advantage is a slightly shorter processing time. The limits of the $2 \mathrm{D}$ CWT technique are related to:

- fringe period: too sparse pattern results in wavelet matching problems; we can avoid it by appropriately tilting the reference beam before recording the images;

- significant deviation of fringes from straightness; processing such an image may introduce errors (this topic is under investigation to optimize the performance of the 2D CWT method).

Two proposed paths of interferogram analysis do not require sophisticated equipment (as the TPS method) and complicated data analysis. Separate information on both surfaces of the object under test and its thickness variations can be obtained with a simple measurement procedure without any interaction into the sample.

Detailed accuracy analyses taking into consideration possible interferometer hardware and software errors are being conducted. We believe that by eliminating or reducing the error sources we can reach one order better accuracy.

\section{References}

[1] P. de Groot, Appl. Opt. 39(16), 2658 (2000).

[2] J. Schwider, Progress in Optics 28 (ed. E. Wolf, North Holland, Amsterdam 1990)

[3] K. Hibino, B.F. Oreb, P.S. Fairman, J. Burke, Appl. Opt. 43(6), 1241 (2004).

[4] K.G. Larkin, D.J. Bone, M.A. Oldfield, J. Opt. Soc. Am. A 18(8) 1862 (2001).

[5] M.A. Gdeisat, D.R. Burton, M.J. Lalor, Appl. Opt. 45(34), 8722 (2006).

[6] C. Ai, J.C. Wyant, Appl. Opt. 27(14), 3039 (1988)

[7] A. Styk, K. Patorski, Proc. SPIE 7063, 70630P (2008).

[8] Z. Wang, H. Ma, Opt. Eng. 45(4), 045601 (2006). 